severe COVID-19 pneumonia improved towards the latter stages of Wave 1, due in part to implementation of evidencebased interventions such as early administration of dexamethasone. We aimed to compare symptom burden and functional outcomes post-hospitalisation in Wave 1 and Wave 2 patients attending severe post-COVID clinic.

Methods Prospective single-centre observational cohort study. Patients admitted with severe COVID-19 pneumonia (admission duration $\geq 48$ hours, oxygen requirement $\geq 40 \%$ or critical care admission) were invited to the severe post-COVID clinic at 6-8 weeks following hospital discharge. Demographics and anthropometrics, inpatient clinical course, patient-reported (symptoms, functional disability, mental health) and physiological outcomes (4-metre gait speed (4MGS), 1-minute sit-tostand (1STS) repetitions and $\mathrm{SpO}_{2}$ desaturation) were recorded. Outcomes from patients admitted during Wave 1 (until 31/8/2020) were compared to patients admitted during Wave 2 (after 01/9/2020).

Results Between June 2020 and April 2021, 167 1st wave (W1) and 141 2nd wave survivors (W2) were assessed in clinic at $62(50-72)$ and $61(58-65)$ days post hospital discharge respectively. Age, gender, ethnicity and multimorbidity was comparable in W1 and W2 (Table 1). Dexamethasone had been administered to $6.6 \%$ of W1 and $97.9 \%$ of W2 patients. Comparing W2 to W1, length of hospital stay was shorter $(10(7-14)$ vs 15 (9-38) days, $\mathrm{p}<0.001$ ), invasive mechanical ventilation (IMV) was less frequent $(22.7 \%$ vs $37.2 \%)$ and IMV duration was shorter (7 (4-12) vs $30(11-40)$ days, $\mathrm{p}<0.001)$. There were no significant differences in breathlessness, cough, pain, fatigue, 4MGS or 1STS $\mathrm{SpO}_{2}$ desaturation (table 1). However, 1STS performance (repetitions/min) was below age and gender-adjusted lower limits of normal $(<2.5$ percentile) in $77.7 \%$ of W2 compared to $60.0 \%$ of W1 patients $(\mathrm{p}=0.011)$.

Conclusion Despite shorter admission duration, and less frequent IMV, the burden of symptoms and functional limitation experienced post-hospitalisation for severe COVID-19 pneumonia was at least as severe during Wave 2 as in Wave 1. Identification of contributing factors and impact on post-COVID rehabilitation outcomes requires further study.

\section{P136 THE RELATIONSHIP BETWEEN SYMPTOMS AND FUNCTIONAL PHYSIOLOGICAL OUTCOMES IN SURVIVORS OF SEVERE COVID-19 PNEUMONIA}

${ }^{1} \mathrm{P}$ Zamani, 'S Mehrotra, ${ }^{1} \mathrm{D}$ Griffin, ${ }^{1} \mathrm{RF} \mathrm{D}^{\prime} \mathrm{Cruz},{ }^{2} \mathrm{~S}$ Mathew, ${ }^{2} \mathrm{~A}$ Byrne, ${ }^{2} \mathrm{M}$ Choudhury, ${ }^{2} \mathrm{~T}$ Fleming, ${ }^{2} \mathrm{P} C \mathrm{Cho},{ }^{2} \mathrm{D}$ Walder, ${ }^{2} \mathrm{R}$ Madula, ${ }^{2} \mathrm{~L}$ Smith, ${ }^{2} \mathrm{P}$ Macedo, ${ }^{3} \mathrm{~S}$ Norton, ${ }^{1,2} \mathrm{~S}$ Birring, ${ }^{1,2} \mathrm{~A}$ Patel, ${ }^{1,2} \mathrm{I}$ Patel, ${ }^{2} \mathrm{~F}$ Perrin, ${ }^{1,2} \mathrm{M}$ Waller, ${ }^{1,2} \mathrm{CJ}$ Jolley. ${ }^{1}$ Centre for Human and Applied Physiological Sciences, King's College London, King's Health Partners, London, UKi ${ }^{2}$ Department of Respiratory Medicine, King's College Hospital NHS Foundation Trust, London, UK; ${ }^{3}$ Centre for Rheumatic Diseases, King's College London, London, UK

\subsection{6/thorax-2021-BTSabstracts.245}

Introduction and Objectives Holistic post-COVID assessment should include physiological testing, however this may be challenging to deliver in accordance with local infection prevention control precautions. The aim of this study was to evaluate associations between patient-reported and physiological measures of recovery following severe COVID-19 pneumonia. The principal objective was to understand whether patient characteristics and symptom scores are predictive of physiological impairment.

Methods This was a prospective single-centre observational study undertaken in the severe COVID-19 follow-up clinic of a South London Hospital. Survivors of severe COVID-19 pneumonia were assessed in clinic at $6-8$ weeks post hospital discharge. Relationships between symptoms (outcome measures are listed in table 1), 4-metre gait speed (4MGS), and 1minute sit-to-stand (repetitions/min (1STSreps) and $\mathrm{SpO}_{2} \%$ desaturation) were assessed by correlation and multiple regression analysis.

Results Between June 2020-March 2021, 311 patients were assessed in clinic. Mean \pm SD age $59 \pm 13,60 \%$ male, median (IQR) BMI $\left.31.4 \quad(27.5-36.3) \quad \mathrm{kg} / \mathrm{m}^{2}\right) . \quad 30 \%$ had received invasive mechanical ventilation, $13 \%$ received high flow nasal oxygen, $8 \%$ received CPAP, $1 \%$ received non-invasive ventilation and $48 \%$ required facemask oxygen (FMO2) only. At follow-up, 39\% breached the clinically-significant threshold for $4 \mathrm{MGS}(4 \mathrm{MGS}<0.8 \mathrm{~m} / \mathrm{s})$ and $66 \%$ breached the theshold for 1STS performance (1STSreps <2.5 percentile adjusted for age and gender). 33\% desaturated $\geq 4 \%$ during 1STS testing.

Abstract P136 Table 1 Results of correlation analysis

\begin{tabular}{|c|c|c|c|c|c|c|c|}
\hline & & \multicolumn{6}{|c|}{ Correlation analysis } \\
\hline & & \multicolumn{2}{|l|}{ 4MGS } & \multicolumn{2}{|l|}{ 1STSreps } & \multicolumn{2}{|c|}{$\mathrm{SpO}_{2} \%$ desaturation } \\
\hline & Results & $\mathbf{r}$ & p-value & $\mathbf{r}$ & p-value & $\mathbf{r}$ & $\begin{array}{l}\mathrm{p} \text { - } \\
\text { value }\end{array}$ \\
\hline Pre-COVID mMRC dyspnoea score & $0(0-1)$ & $-0.267^{* *}$ & $<0.001$ & $-0.285^{* *}$ & $<0.001$ & -0.108 & 0.094 \\
\hline $\begin{array}{l}\text { Post-COVID mMRC dyspnoea } \\
\text { score }\end{array}$ & $1(0-2)$ & -0.442 ** & $<0.001$ & $-0.457^{* *}$ & $<0.001$ & $-0.143^{*}$ & 0.025 \\
\hline NRS breathlessness & $3(0-5)$ & $-0.287^{* *}$ & $<0.001$ & $-0.406^{* *}$ & $<0.001$ & -0.490 & 0.445 \\
\hline NRS fatigue & $3(0-5)$ & $-0.315^{* *}$ & $<0.001$ & $-0.379^{* *}$ & $<0.001$ & $-0.190^{*}$ & 0.003 \\
\hline NRS cough & $0(0-2)$ & -0.660 & 0.292 & $-0.153^{*}$ & 0.017 & 0.083 & 0.194 \\
\hline NRS pain & $1(0-4)$ & $-0.278^{* *}$ & $<0.001$ & $-0.346^{\star *}$ & $<0.001$ & $-0.188^{*}$ & 0.003 \\
\hline NRS sleep difficulty & $2(0-5)$ & $-0.246^{* *}$ & $<0.001$ & $-0.386^{* *}$ & $<0.001$ & -0.122 & 0.057 \\
\hline
\end{tabular}

Data are presented as median (interquartile range) or frequency (proportion $\% ; 95 \%$ confidence interval). $\mathrm{SpO}_{2} \%$ desaturation $=\mathrm{SpO}_{2} \%$ desaturation from baseline during 1 minute sit to stand test; 1 STSreps = repetitions per minute during 1 minute sit to stand test; $4 \mathrm{MGS}=4$ metre gait speed; $\mathrm{mMRC}=$ modified Medical Research Council; NRS $=0-10$ numerical rating scale; $r=$ Spearman correlation coefficient. *indicates statistical significance at 0.05 level. **indicates statistical significance at 0.001 level. 
Correlations between symptoms and outcomes of physiological tests are shown in table 1 . Age (beta=-0.007, p<0.001), male gender (beta $=0.073, \mathrm{p}=035$ ), current mMRC dyspnoea score (beta $=-0.107, \mathrm{p}<0.001$ ) and pain (beta $=-0.017, \mathrm{p}$ 0.020 ) were independent predictors of 4MGS (overall model fit $\left.\mathrm{R}^{2}=0.394, \mathrm{p}<0.001\right)$. Current mMRC dyspnoea score (beta $=-1.274, \mathrm{p}=0.019$ ) and use of facemask $\mathrm{O}_{2}$ as maximal inpatient respiratory support (beta $=2.188, \mathrm{p}=0.012$ ) were independent predictors of 1STSreps $\left(\mathrm{R}^{2}=0.309, \mathrm{p}<0.001\right)$. Variation in $\mathrm{SpO}_{2} \%$ desaturation during the 1STS was not explained by the regression model $\left(\mathrm{R}^{2}=0.078, \mathrm{p}=0.082\right)$.

Conclusion Respiratory symptoms were not strong predictors of 4-metre gait speed and 1-minute sit-to-stand test performance. These data highlight the importance of face-to-face testing to objectively assess functional limitation in patients recovering from severe COVID pneumonia.

\section{P137 THE IMPACT OF ETHNICITY ON THE LONG-TERM SEQUELAE OF COVID-19: FOLLOW-UP FROM THE FIRST AND SECOND WAVES IN NORTH LONDON}

${ }^{1}$ SB Naidu, ${ }^{1} \mathrm{AJ}$ Shah, ${ }^{1} \mathrm{~A}$ Saigal, ${ }^{1} \mathrm{SE}$ Brill, ${ }^{1} \mathrm{H}$ Jarvis, ${ }^{1} \mathrm{JG}$ Goldring, ${ }^{1} \mathrm{E}$ Wey, ${ }^{2} \mathrm{D}$ Miller, ${ }^{3}$ I Abubakar, ${ }^{1,2} \mathrm{JR}$ Hurst, ${ }^{1,2} \mathrm{M}$ Lipman, 'S Mandal. 'Royal Free London NHS Trust, London, UK; ${ }^{2}$ Open Health UK, London, UK; ${ }^{3}$ UCL Respiratory, London, UK

\subsection{6/thorax-2021-BTSabstracts.246}

Introduction Ethnicity has been reported as a possible risk factor for SARS-CoV-2 infection, with one meta-analysis suggesting individuals from Black, Asian and other minority ethnic (BAME) backgrounds are at increased risk of infection but not necessarily severe infection or death ${ }^{1}$. However, there is little information on whether such individuals are more likely to develop prolonged symptoms after infection or 'Long COVID'.

Methods We established a follow-up clinic to review all individuals admitted to our Trust with COVID-19 and collected data on demographics, symptom burden and mental health outcomes at 8-10 weeks post discharge. We categorised our cohort into four main groups (White, Black, Asian and Other) based on self-reported ethnicity.

Results Between 27/2/20 and 17/2/21, our Trust admitted 2217 adults with COVID-19. Ethnicity data were available for 1806 individuals; 52.9\% were White, 16.3\% Asian, $12.5 \%$ Black and $18.3 \%$ from Other ethnic backgrounds. 342 patients died during admission; 63.2\% were White, 9.4\% Asian, $11.7 \%$ Black and $15.8 \%$ from Other ethnic backgrounds. More White adults died than expected $(\mathrm{p}<0.001)$.

Follow-up was completed on 1107 of 1464 (75.6\%) adults discharged. 54.5\% were White, 22.8\% Asian, $11.7 \%$ Black and $11.0 \%$ from Other ethnic backgrounds. Table 1 summarises key demographic, admission and follow-up symptoms. Adults from White backgrounds were older and less likely to have diabetes $(\mathrm{p}<0.001)$, while Black adults were more likely to have higher BMI, hypertension and chronic kidney disease $(p<0.002)$. There was no difference in admission National Early Warning Score (NEWS2), or number of adults requiring non-invasive respiratory support and intubation.

At follow up, there was no difference between ethnic groups in terms of burden of physical or mental health symptoms, breathlessness score and ability to return to work.
Abstract P137 Table 1 Demographics, admission severity and follow-up symptoms

\begin{tabular}{|c|c|c|c|c|c|}
\hline Variable & $\begin{array}{l}\text { White } \\
N=603\end{array}$ & $\begin{array}{l}\text { Asian } \\
\mathrm{N}=252\end{array}$ & $\begin{array}{l}\text { Black } \\
\mathrm{N}=130\end{array}$ & $\begin{array}{l}\text { Other } \\
\mathrm{N}=122\end{array}$ & $\mathrm{p}$-value \\
\hline Age & $\begin{array}{l}65 \pm \\
16.5\end{array}$ & $\begin{array}{l}59 \pm \\
15.4\end{array}$ & $\begin{array}{l}59 \pm \\
13.9\end{array}$ & $\begin{array}{l}59 \pm \\
14.7\end{array}$ & $<0.001$ \\
\hline Male (\%) & $372(62)$ & $148(59)$ & $72(55)$ & $81(66)$ & 0.28 \\
\hline Index of deprivation* & $6(3-7)$ & $6(4-8)$ & $5(3-7)$ & $5(3-7)$ & 0.03 \\
\hline Body mass index & $\begin{array}{l}27.1 \\
(23.5- \\
30.5)\end{array}$ & $\begin{array}{l}26.0 \\
(23.7- \\
29.2)\end{array}$ & $\begin{array}{l}28.9 \\
(25.9- \\
34.7)\end{array}$ & $\begin{array}{l}26.7 \\
(25.1- \\
30.9)\end{array}$ & $<0.001$ \\
\hline Hypertension (\%) & $\begin{array}{l}244 / 583 \\
(42)\end{array}$ & $\begin{array}{l}113 / 247 \\
(46)\end{array}$ & $\begin{array}{l}72 / 125 \\
(58)\end{array}$ & $\begin{array}{l}40 / 119 \\
(34)\end{array}$ & 0.001 \\
\hline Cardiovascular disease (\%) & $\begin{array}{l}133 / 415 \\
(32)\end{array}$ & $\begin{array}{l}45 / 166 \\
(27)\end{array}$ & $\begin{array}{l}15 / 86 \\
(17)\end{array}$ & $\begin{array}{l}16 / 73 \\
(22)\end{array}$ & 0.02 \\
\hline Diabetes (\%) & $\begin{array}{l}122 / 583 \\
(21)\end{array}$ & $\begin{array}{l}80 / 247 \\
(32)\end{array}$ & $\begin{array}{l}45 / 125 \\
(36)\end{array}$ & $\begin{array}{l}40 / 119 \\
(34)\end{array}$ & $<0.001$ \\
\hline Respiratory disease (\%) & $\begin{array}{l}124 / 416 \\
(30)\end{array}$ & $\begin{array}{l}39 / 167 \\
(23)\end{array}$ & $\begin{array}{l}21 / 91 \\
(23)\end{array}$ & $\begin{array}{l}16 / 70 \\
(23)\end{array}$ & 0.25 \\
\hline Chronic kidney disease (\%) & $\begin{array}{l}61 / 583 \\
(11)\end{array}$ & $\begin{array}{l}29 / 247 \\
(12)\end{array}$ & $\begin{array}{l}27 / 125 \\
(22)\end{array}$ & 9/119 (8) & 0.002 \\
\hline Any mental health (\%) & $\begin{array}{l}77 / 583 \\
(13)\end{array}$ & $\begin{array}{l}23 / 247 \\
(9)\end{array}$ & $8 / 125(6)$ & $\begin{array}{l}17 / 119 \\
(14)\end{array}$ & 0.08 \\
\hline Smoking history (\%) & $\begin{array}{l}250 / 587 \\
(43)\end{array}$ & $\begin{array}{l}43 / 244 \\
(18)\end{array}$ & $\begin{array}{l}44 / 127 \\
(35)\end{array}$ & $\begin{array}{l}44 / 118 \\
(37)\end{array}$ & $<0.001$ \\
\hline Clinical frailty score & $3(2-4)$ & $2(2-4)$ & $3(2-4)$ & $3(2-3)$ & 0.11 \\
\hline NEWS2 & $4(2-6)$ & $4.5(3-6)$ & $5(3-6)$ & $5(3-7)$ & 0.3 \\
\hline Respiratory support & $\begin{array}{l}80 / 564 \\
(14)\end{array}$ & $\begin{array}{l}47 / 236 \\
(20)\end{array}$ & $\begin{array}{l}23 / 116 \\
(20)\end{array}$ & $\begin{array}{l}21 / 118 \\
(18)\end{array}$ & 0.15 \\
\hline Follow-up symptoms & & & & & \\
\hline MRC score* & $2(1-3)$ & $2(1-3)$ & $2(1-3)$ & $2(1-2)$ & 0.61 \\
\hline Cough improved (\%) & $\begin{array}{l}414 / 553 \\
(75)\end{array}$ & $\begin{array}{l}177 / 232 \\
(76)\end{array}$ & $\begin{array}{l}85 / 118 \\
(72)\end{array}$ & $\begin{array}{l}87 / 113 \\
(77)\end{array}$ & 0.8 \\
\hline Fatigue improved (\%) & $\begin{array}{l}451 / 552 \\
(82)\end{array}$ & $\begin{array}{l}193 / 228 \\
(85)\end{array}$ & $\begin{array}{l}109 / 122 \\
(89)\end{array}$ & $\begin{array}{l}88 / 115 \\
(77)\end{array}$ & 0.05 \\
\hline Sleep improved (\%) & $\begin{array}{l}342 / 536 \\
(64)\end{array}$ & $\begin{array}{l}155 / 228 \\
(68)\end{array}$ & $\begin{array}{l}76 / 117 \\
(65)\end{array}$ & $\begin{array}{l}68 / 112 \\
(61)\end{array}$ & 0.57 \\
\hline Burden of symptoms* & $1(0-2)$ & $1(0-2)$ & $1(0-2)$ & $1(0-2)$ & 0.78 \\
\hline Returned to work (\%) & $\begin{array}{l}142 / 256 \\
(56)\end{array}$ & $\begin{array}{l}77 / 139 \\
(55)\end{array}$ & $\begin{array}{l}29 / 64 \\
(45)\end{array}$ & $\begin{array}{l}41 / 71 \\
(58)\end{array}$ & 0.45 \\
\hline Felt back to normal (\%) & $\begin{array}{l}256 / 439 \\
(55)\end{array}$ & $\begin{array}{l}112 / 186 \\
(60)\end{array}$ & $\begin{array}{l}62 / 103 \\
(60)\end{array}$ & $\begin{array}{l}43 / 78 \\
(55)\end{array}$ & 0.87 \\
\hline $\begin{array}{l}\text { Positive PHQ-2 (depression } \\
\text { screening) }\end{array}$ & $\begin{array}{l}65 / 580 \\
(11)\end{array}$ & $\begin{array}{l}40 / 242 \\
(17)\end{array}$ & $\begin{array}{l}12 / 126 \\
(10)\end{array}$ & $\begin{array}{l}11 / 116 \\
(10)\end{array}$ & 0.09 \\
\hline $\begin{array}{l}\text { Positive TSQ (post-traumatic } \\
\text { stress screening) }\end{array}$ & $\begin{array}{l}42 / 601 \\
(7)\end{array}$ & $\begin{array}{l}16 / 248 \\
(7)\end{array}$ & $\begin{array}{l}13 / 130 \\
(10)\end{array}$ & $8 / 122(7)$ & 0.6 \\
\hline
\end{tabular}

*Non-parametric data presented as median \pm interquartile range, all other data presented as mean \pm standard deviation.

Discussion Our data demonstrate that despite having more comorbidities associated with worse outcomes, adults from BAME backgrounds who are discharged from hospital following COVID-19 are no more likely to experience symptoms consistent with 'Long COVID'. However, given the increased risk of infection among BAME communities, we must ensure that reducing health inequalities remain central to the UK health agenda.

\section{REFERENCE}

1. Sze, et al. EClinica/Medicine 2020. doi:10.1016/.eclinm.2020.100630 